\title{
Global nonexistence of solutions for systems of quasilinear hyperbolic equations with damping and source terms
}

Yaojun Ye

\section{"Correspondence:} yjye2013@163.com Department of Mathematics and Information Science, Zhejiang University of Science and Technology, Hangzhou, 310023, People's Republic of China

\begin{abstract}
The initial boundary value problem for a class of quasilinear hyperbolic equations system in a bounded domain is studied. We prove that the solutions with positive initial energy blow up in finite time under some conditions. The estimates of the lifespan of solutions are given.

MSC: 35A05; 35B40; 35L80
\end{abstract}

Keywords: nonlinear hyperbolic equations system; global solutions; lifespan of solutions

\section{Introduction}

In this paper, we are concerned with the blow-up of solutions for the following quasilinear hyperbolic equations system:

$$
\begin{aligned}
& u_{t t}-\operatorname{div}\left(|\nabla u|^{m-2} \nabla u\right)+a\left|u_{t}\right|^{p-2} u_{t}-\Delta u_{t}=f_{1}(u, v), \quad(x, t) \in \Omega \times R^{+}, \\
& v_{t t}-\operatorname{div}\left(|\nabla v|^{m-2} \nabla v\right)+a\left|v_{t}\right|^{p-2} v_{t}-\Delta v_{t}=f_{2}(u, v), \quad(x, t) \in \Omega \times R^{+}
\end{aligned}
$$

with the initial boundary value conditions

$$
\begin{aligned}
& u(x, 0)=u_{0}(x) \in W_{0}^{1, m}(\Omega), \quad u_{t}(x, 0)=u_{1}(x) \in L^{2}(\Omega), \quad x \in \Omega, \\
& v(x, 0)=v_{0}(x) \in W_{0}^{1, m}(\Omega), \quad v_{t}(x, 0)=v_{1}(x) \in L^{2}(\Omega), \quad x \in \Omega, \\
& u(x, t)=0, \quad v(x, t)=0, \quad(x, t) \in \partial \Omega \times R^{+},
\end{aligned}
$$

where $\Omega$ is a bounded open domain in $R^{n}$ with a smooth boundary $\partial \Omega . a>0$ and $m, p \geq 2$ are real numbers, and $f_{i}(\cdot, \cdot): R^{2} \rightarrow R(i=1,2)$ are given functions to be determined later.

When $m=2$, problem (1.1)-(1.5) defines the motion of charged meson in an electromagnetic field and was proposed by Segal [1]. Equations (1.1) and (1.2) with initial boundary conditions (1.3)-(1.5), but without dissipative terms, were early considered by several authors. Medeiros and Miranda [2,3] showed the existence and uniqueness of global weak solutions. Da Silva Ferreira [4] proved that the first-order energy decays exponentially in the presence of frictional local damping. Cavalcanti et al. [5] considered the asymptotic 
behavior for an analogous hyperbolic-parabolic system, with boundary damping, using arguments from Komornik and Zuazua [6].

For the initial boundary value problem of a single quasilinear hyperbolic equation

$$
\begin{aligned}
& u_{t t}-\operatorname{div}\left(|\nabla u|^{m-2} \nabla u\right)+a\left|u_{t}\right|^{p-2} u_{t}-\Delta u_{t}=b|u|^{r-2} u, \quad(x, t) \in \Omega \times R^{+}, \\
& u(x, 0)=u_{0}(x) \in W_{0}^{1, m}(\Omega), \quad u_{t}(x, 0)=u_{1}(x) \in L^{2}(\Omega), \quad x \in \Omega, \\
& u(x, t)=0, \quad(x, t) \in \partial \Omega \times R^{+},
\end{aligned}
$$

Yang and Chen [7-9] studied problem (1.6)-(1.8) and obtained global existence results under the growth assumptions on the nonlinear terms and the initial value. These global existence results have been improved by Liu and Zhao [10] by using a new method. In [11], the author considered a similar problem to (1.6)-(1.8) and proved a blow-up result under the condition $r>\max \{p, m\}$ and that the initial energy is sufficiently negative. Messaoudi and Said-Houari [12] improved the results in [11] and showed that the blow-up takes place for negative initial data only regardless of the size of $\Omega$. By means of the perturbed energy and the potential well methods, Messaoudi [13] gave precise decay rates for the solution of problem (1.6)-(1.8). In particular, he showed that for $m=2$, the decay is exponential.

In absence of the strong damping $-\Delta u_{t}$, equation (1.6) becomes

$$
u_{t t}-\operatorname{div}\left(|\nabla u|^{m-2} \nabla u\right)+a\left|u_{t}\right|^{p-2} u_{t}=b|u|^{r-2} u, \quad(x, t) \in \Omega \times R^{+} .
$$

For $b=0$, it is well known that the damping term assures global existence and decay of the solution energy for arbitrary initial value (see [14-16]). For $a=0$, the source term causes finite time blow-up of solutions with negative initial energy if $r>m$ (see [17]). When the quasilinear operator $-\operatorname{div}\left(|\nabla u|^{m-2} \nabla u\right)$ is replaced by $\Delta^{2} u$, Wu and Tsai [18] showed that the solution is global in time under some conditions without the relation between $p$ and $r$. They also proved that the local solution blows up in finite time if $r>p$ and the initial energy is nonnegative, and gave the decay estimates of the energy function and the lifespan of solutions.

In this paper we show that the local solutions of problem (1.1)-(1.5) with small positive initial energy blow up in finite time. Meanwhile, the lifespan of solutions is given. The main tool of the proof is a technique introduced by paper [19] and some estimates used firstly by Vitillaro [20] in order to study a class of single wave equations.

For simplicity of notations, hereafter we denote by $\|\cdot\|_{s}$ the space $L^{s}(\Omega)$ norm, $\|\cdot\|$ denotes $L^{2}(\Omega)$ norm, and we write an equivalent norm $\|\nabla \cdot\|_{m}$ instead of $W_{0}^{1, m}(\Omega)$ norm $\|\cdot\|_{W_{0}^{1, m}(\Omega)}$. Moreover, $C$ denotes various positive constants depending on the known constants and may be different at each appearance.

\section{Preliminaries}

Concerning the functions $f_{1}(u, v)$ and $f_{2}(u, v)$, we assume that

$$
\begin{aligned}
& f_{1}(u, v)=b_{1}|u+v|^{2 r}(u+v)+b_{2}|u|^{r-1} u|v|^{r+1}, \\
& f_{2}(u, v)=b_{1}|u+v|^{2 r}(u+v)+b_{2}|v|^{r-1} v|u|^{r+1},
\end{aligned}
$$

where $b_{1}, b_{2}>0$ and $r>0$ are constants. 
It is easy to see that

$$
u f_{1}(u, v)+v f_{2}(u, v)=2(r+1) F(u, v), \quad \forall(u, v) \in R^{2}
$$

where

$$
F(u, v)=\frac{b_{1}}{2(r+1)}|u+v|^{2(r+1)}+\frac{b_{2}}{r+1}|u v|^{r+1} .
$$

Moreover, a quick computation will show that there exist two positive constants $C_{0}$ and $C_{1}$ such that the following inequality holds (see [21]):

$$
\frac{C_{0}}{2(r+1)}\left(|u|^{2(r+1)}+|v|^{2(r+1)}\right) \leq F(u, v) \leq \frac{C_{1}}{2(r+1)}\left(|u|^{2(r+1)}+|v|^{2(r+1)}\right) .
$$

Now, we define the following energy function associated with a solution $[u, v]$ of problem (1.1)-(1.5):

$$
E(t)=\frac{1}{2}\left(\left\|u_{t}(t)\right\|^{2}+\left\|v_{t}(t)\right\|^{2}\right)+\frac{1}{m}\left(\|\nabla u(t)\|_{m}^{m}+\|\nabla v(t)\|_{m}^{m}\right)-\int_{\Omega} F(u, v) d x
$$

for $[u, v] \in W_{0}^{1, m}(\Omega) \times W_{0}^{1, m}(\Omega)$, and

$$
E(0)=\frac{1}{2}\left(\left\|u_{1}\right\|^{2}+\left\|v_{1}\right\|^{2}\right)+\frac{1}{m}\left(\left\|\nabla u_{0}\right\|_{m}^{m}+\left\|\nabla v_{0}\right\|_{m}^{m}\right)-\int_{\Omega} F\left(u_{0}, v_{0}\right) d x
$$

is the initial total energy.

Note that we have from (2.5) that

$$
E(t) \geq \frac{1}{m}\left(\|\nabla u(t)\|_{m}^{m}+\|\nabla v(t)\|_{m}^{m}\right)-\int_{\Omega} F(u, v) d x
$$

for $[u, v] \in W_{0}^{1, m}(\Omega) \times W_{0}^{1, m}(\Omega)$.

Lemma 2.1 Let $s$ be a number with $2 \leq s<+\infty$ if $n \leq m$ and $2 \leq s \leq \frac{n m}{n-m}$ if $n>m$. Then there is a constant $C$ depending on $\Omega$ and s such that

$$
\|u\|_{s} \leq C\|\nabla u\|_{m}, \quad \forall u \in W_{0}^{1, m}(\Omega) .
$$

Lemma 2.2 (Young's inequality) Let $a, b \geq 0$ and $\frac{1}{p}+\frac{1}{q}=1$ for $1<p, q<+\infty$, then one has the inequality

$$
a b \leq \delta a^{p}+C(\delta) b^{q}
$$

where $\delta>0$ is an arbitrary constant, and $C(\delta)$ is a positive constant depending on $\delta$.

We get from Minkowski's inequality and Lemma 2.1 that

$$
\|u+v\|_{2(r+1)}^{2} \leq 2\left(\|u\|_{2(r+1)}^{2}+\|v\|_{2(r+1)}^{2}\right) \leq 2 B^{2}\left(\|\nabla u\|_{m}^{2}+\|\nabla v\|_{m}^{2}\right) .
$$


Also, we have from Hölder's inequality and Lemma 2.2 that

$$
\begin{aligned}
\|u v\|_{r+1} & \leq\|u(t)\|_{2(r+1)} \cdot\|v(t)\|_{2(r+1)} \leq \frac{1}{2}\left(\|u(t)\|_{2(r+1)}^{2}+\|v(t)\|_{2(r+1)}^{2}\right) \\
& \leq \frac{B^{2}}{2}\left(\|\nabla u\|_{m}^{2}+\|\nabla v\|_{m}^{2}\right)
\end{aligned}
$$

where $B$ is the optimal Sobolev constant from $W_{0}^{1, m}(\Omega)$ to $L^{2(r+1)}(\Omega)$.

We get from (2.3), (2.8) and (2.9) that

$$
\int_{\Omega} F(u, v) d x \leq \frac{C_{2} B^{2(r+1)}}{r+1}\left(\|\nabla u\|_{m}^{2}+\|\nabla v\|_{m}^{2}\right)^{r+1},
$$

where $C_{2}=2^{r} b_{1}+\frac{b_{2}}{2^{r+1}}$.

Considering the basic inequality $|x+y|^{\rho} \leq 2^{\rho-1}\left(|x|^{\rho}+|y|^{\rho}\right), \forall x, y \in R, \rho \geq 1$, it follows from (2.7) and (2.10) that

$$
\begin{aligned}
E(t) & \geq \frac{1}{m 2^{\frac{m-2}{2}}}\left(\|\nabla u\|_{m}^{2}+\|\nabla v\|_{m}^{2}\right)^{\frac{m}{2}}-\frac{C_{2} B^{2(r+1)}}{r+1}\left(\|\nabla u\|_{m}^{2}+\|\nabla v\|_{m}^{2}\right)^{r+1} \\
& =Q\left(\sqrt{\|\nabla u\|_{m}^{2}+\|\nabla v\|_{m}^{2}}\right)
\end{aligned}
$$

where

$$
Q(\lambda)=\frac{1}{m 2^{\frac{m-2}{2}}} \lambda^{m}-\frac{C_{2} B^{2(r+1)}}{r+1} \lambda^{2(r+1)} .
$$

Therefore, we get that

$$
\begin{aligned}
& Q^{\prime}(\lambda)=\frac{1}{2^{\frac{m-2}{2}}} \lambda^{m-1}-2 C_{2} B^{2(r+1)} \lambda^{2 r+1}, \\
& Q^{\prime \prime}(\lambda)=\frac{m-1}{2^{\frac{m-2}{2}}} \lambda^{m-2}-2(2 r+1) C_{2} B^{2(r+1)} \lambda^{2 r} .
\end{aligned}
$$

Let $Q^{\prime}(\lambda)=0$, which implies that $\lambda_{1}=\left(2^{\frac{m}{2}} C_{2} B^{2(r+1)}\right)^{\frac{1}{m-2(r+1)}}$. As $\lambda=\lambda_{1}$ and $m<2(r+1)$, an elementary calculation shows that

$$
Q^{\prime \prime}(t)=\frac{m-2(r+1)}{2^{\frac{m-2}{2}}}\left(2^{\frac{m}{2}} C_{2} B^{2(r+1)}\right)^{\frac{m-2}{m-2(r+1)}}<0 .
$$

Thus, $Q(\lambda)$ has the maximum at $\lambda_{1}$ and the maximum value is

$$
d=Q\left(\lambda_{1}\right)=\frac{1}{2^{\frac{m-2}{2}}}\left(\frac{1}{m}-\frac{1}{2(r+1)}\right)\left(2^{\frac{m}{2}} C_{2} B^{2(r+1)}\right)^{\frac{m}{m-2(r+1)}} .
$$

In order to prove our main result, we need the following two lemmas.

Lemma 2.3 Let $[u, v]$ be a solution to problem (1.1)-(1.5), then $E(t)$ is a nonincreasing function for $t>0$ and

$$
\frac{d}{d t} E(t)=-\left(a\left\|u_{t}\right\|_{p}^{p}+a\left\|v_{t}\right\|_{p}^{p}+\left\|\nabla u_{t}\right\|_{2}^{2}+\left\|\nabla v_{t}\right\|_{2}^{2}\right) \leq 0
$$


Multiplying equation (1.1) by $u_{t}$ and (1.2) by $v_{t}$, and integrating over $\Omega \times[0, t]$, then adding them together and integrating by parts, we get

$$
E(t)-E(0)=-\int_{0}^{t}\left(a\left\|u_{t}(s)\right\|_{p}^{p}+a\left\|v_{t}(s)\right\|_{p}^{p}+\left\|\nabla u_{t}(s)\right\|_{2}^{2}+\left\|\nabla v_{t}(s)\right\|_{2}^{2}\right) d s
$$

for $t \geq 0$.

Being the primitive of an integrable function, $E(t)$ is absolutely continuous and equality (2.13) is satisfied.

Applying the idea of Vitillaro [20], we have the following lemma.

Lemma 2.4 Assume that $0<E(0)<d$.

(i) If $\left(\left\|\nabla u_{0}\right\|_{m}^{2}+\left\|\nabla v_{0}\right\|_{m}^{2}\right)^{\frac{m}{2}}<\lambda_{1}^{m}$, then $\left(\|\nabla u\|_{m}^{2}+\|\nabla v\|_{m}^{2}\right)^{\frac{m}{2}}<\lambda_{1}^{m}$ for $t \geq 0$.

(ii) If $\left(\left\|\nabla u_{0}\right\|_{m}^{2}+\left\|\nabla v_{0}\right\|_{m}^{2}\right)^{\frac{m}{2}}>\lambda_{1}^{m}$, then there exists $\lambda_{2}>\lambda_{1}$ such that $\left(\|\nabla u\|_{m}^{2}+\|\nabla v\|_{m}^{2}\right)^{\frac{m}{2}} \geq \lambda_{2}^{m}$ for $t \geq 0$.

For the detailed proof of Lemma 2.4, one can refer to [18].

We conclude this section by stating the local existence and uniqueness of solutions for problem (1.1)-(1.5), which can be obtained by a similar way as done in $[7-9,22]$. The result reads as follows.

Theorem 2.1 (Local solution) Suppose that $\left[u_{0}, v_{0}\right] \in W_{0}^{1, m}(\Omega) \times W_{0}^{1, m}(\Omega),\left[u_{1}, v_{1}\right] \in$ $L^{2}(\Omega) \times L^{2}(\Omega)$ and

$$
2<m<2(r+1) \leq \frac{n m}{n-m}, \quad n \geq m ; \quad 2<m<2(r+1)<+\infty, \quad n<m,
$$

then there exists $T>0$ such that problem (1.1)-(1.5) has a unique local solution $[u(t), v(t)]$ satisfying

$$
\begin{aligned}
& {[u, v] \in L^{\infty}\left([0, T) ; W_{0}^{1, m}(\Omega) \times W_{0}^{1, m}(\Omega)\right),} \\
& u_{t}, v_{t} \in L^{\infty}\left([0, T) ; L^{2}(\Omega)\right) \cap L^{p}(\Omega \times[0, T)) \cap L^{2}\left([0, T) ; H_{0}^{1}(\Omega)\right) .
\end{aligned}
$$

Moreover, at least one of the following statements holds true:

(1) $\left\|u_{t}\right\|^{2}+\left\|v_{t}\right\|^{2}+\|\nabla u\|_{m}^{m}+\|\nabla v\|_{m}^{m} \rightarrow+\infty$ as $t \rightarrow T^{-}$;

(2) $T=+\infty$.

\section{Main result and proof}

In this section, we prove that the solutions with positive initial energy blow up in finite time under some conditions and that the estimates of the lifespan of solutions are given. Our main result reads as follows.

Theorem 3.1 Assume that (2.15) holds, $r>\frac{1}{2} \max \{p, m\}-1$ and $\left[u_{0}, v_{0}\right] \in W_{0}^{1, m}(\Omega) \times$ $W_{0}^{1, m}(\Omega),\left[u_{1}, v_{1}\right] \in L^{2}(\Omega) \times L^{2}(\Omega)$. If $0<E(0)<d$ and $\left(\left\|\nabla u_{0}\right\|_{m}^{2}+\left\|\nabla v_{0}\right\|_{m}^{2}\right)^{\frac{m}{2}}>\lambda_{1}^{m}$, then the local solution of problem (1.1)-(1.5) blows up in finite time.

Proof Let

$$
H(t)=h-E(t), \quad t \geq 0,
$$


where $h=\frac{E(0)+d}{2}$. We see from (2.13) in Lemma 2.3 that $H^{\prime}(t) \geq 0$. Thus we obtain

$$
H(t) \geq H(0)=h-E(0)>0, \quad t \geq 0 .
$$

Let

$$
G(t)=\int_{\Omega}\left(u u_{t}+v v_{t}\right) d x+\frac{1}{2}\left(\|\nabla u(t)\|^{2}+\|\nabla v(t)\|^{2}\right) .
$$

By differentiating both sides of (3.3) on $t$, we get from (1.1) and (1.2) that

$$
\begin{aligned}
G^{\prime}(t)= & \left(\left\|u_{t}\right\|^{2}+\left\|v_{t}\right\|^{2}\right)-\left(\|\nabla u\|_{m}^{m}+\|\nabla v\|_{m}^{m}\right) \\
& +2(r+1) \int_{\Omega} F(u, v) d x-a \int_{\Omega}\left(\left|u_{t}\right|^{p-2} u_{t} u+\left|v_{t}\right|^{p-2} v_{t} v\right) d x
\end{aligned}
$$

We have from (2.5), (3.1) and (3.4) that

$$
\begin{aligned}
G^{\prime}(t)= & (r+2)\left(\left\|u_{t}\right\|^{2}+\left\|v_{t}\right\|^{2}\right)+\frac{2(r+1)-m}{m}\left(\|\nabla u\|_{m}^{m}+\|\nabla v\|_{m}^{m}\right) \\
& -a \int_{\Omega}\left(\left|u_{t}\right|^{p-2} u_{t} u+\left|v_{t}\right|^{p-2} v_{t} v\right) d x+2(r+1) H(t)-2(r+1) h .
\end{aligned}
$$

We obtain from Lemma 2.4 that

$$
\begin{aligned}
& \frac{2(r+1)-m}{m}\left(\|\nabla u\|_{m}^{m}+\|\nabla v\|_{m}^{m}\right)-2(r+1) h \\
& \geq \frac{2(r+1)-m}{m} \cdot \frac{\left(\lambda_{2}^{m}-\lambda_{1}^{m}\right)}{\lambda_{2}^{m}} \cdot\left(\|\nabla u\|_{m}^{2}+\|\nabla v\|_{m}^{2}\right)^{\frac{m}{2}} \\
& \quad+\frac{2(r+1)-m}{m} \lambda_{1}^{m} \cdot \frac{\left(\|\nabla u\|_{m}^{2}+\|\nabla v\|_{m}^{2}\right)^{\frac{m}{2}}}{\lambda_{2}^{m}}-2(r+1) h \\
& \geq \frac{2(r+1)-m}{m} \cdot \frac{\left(\lambda_{2}^{m}-\lambda_{1}^{m}\right)}{\lambda_{2}^{m}} \cdot\left(\|\nabla u\|_{m}^{2}+\|\nabla v\|_{m}^{2}\right)^{\frac{m}{2}} \\
& \quad+\frac{2(r+1)-m}{m} \lambda_{1}^{m}-2(r+1) h .
\end{aligned}
$$

We have from Lemma 2.4 that $\frac{2(r+1)-m}{m} \cdot \frac{\left(\lambda_{2}^{m}-\lambda_{1}^{m}\right)}{\lambda_{2}^{m}}>0$, and by $(2.12)$ and (3.2), we see that

$$
\begin{aligned}
\frac{2(r+1)-m}{m} \lambda_{1}^{2}-2(r+1) h & =\frac{2(r+1)-m}{m}\left(2^{\frac{m}{2}} C_{2} B^{2(r+1)}\right)^{\frac{2}{m-2(r+1)}}-2(r+1) h \\
& =2(r+1)(d-h)=2(r+1)(h-E(0))>0 .
\end{aligned}
$$

It follows from (3.5), (3.6) and (3.7) that

$$
\begin{aligned}
G^{\prime}(t) \geq & (r+2)\left(\left\|u_{t}\right\|^{2}+\left\|v_{t}\right\|^{2}\right)+\frac{2(r+1)-m}{m} \cdot \frac{\left(\lambda_{2}^{m}-\lambda_{1}^{m}\right)}{\lambda_{2}^{m}} \cdot\left(\|\nabla u\|_{m}^{2}+\|\nabla v\|_{m}^{2}\right)^{\frac{m}{2}} \\
& -a \int_{\Omega}\left(\left|u_{t}\right|^{p-2} u_{t} u+\left|v_{t}\right|^{p-2} v_{t} v\right) d x+2(r+1) H(t) .
\end{aligned}
$$


We have from Hölder's inequality that

$$
\begin{aligned}
& a\left|\int_{\Omega}\left(\left|u_{t}\right|^{p-2} u_{t} u+\left|v_{t}\right|^{p-2} v_{t} v\right) d x\right| \\
& \quad \leq C_{2}\left(\|u\|_{2(r+1)}^{1-\frac{2(r+1)}{p}}\|u\|_{2(r+1)}^{\frac{2(r+1)}{p}}\left\|u_{t}\right\|_{p}^{p-1}+\|v\|_{2(r+1)}^{1-\frac{2(r+1)}{p}}\|v\|_{2(r+1)}^{\frac{2(r+1)}{p}}\left\|v_{t}\right\|_{p}^{p-1}\right) .
\end{aligned}
$$

We get from (2.7), (3.1) and Lemma 2.4 that

$$
\begin{aligned}
H(t) & \leq h-\frac{1}{m 2^{\frac{m-2}{2}}}\left(\|\nabla u\|_{m}^{2}+\|\nabla v\|_{m}^{2}\right)^{\frac{m}{2}}+\int_{\Omega} F(u, v) d x \\
& \leq d-\frac{1}{m 2^{\frac{m-2}{2}}} \lambda_{1}^{m}+\int_{\Omega} F(u, v) d x .
\end{aligned}
$$

Since

$$
d-\frac{1}{m 2^{\frac{m-2}{2}}} \lambda_{1}^{m}=-\frac{1}{2^{\frac{m}{2}}(r+1)}\left(2^{\frac{m}{2}} C_{2} B^{2(r+1)}\right)^{\frac{m}{m-2(r+1)}}<0,
$$

so we have from (3.2), (3.10), (3.11), (2.4) and Lemma 2.1 that

$$
\begin{aligned}
0 & <H(0) \leq H(t) \leq \int_{\Omega} F(u, v) d x \\
& \leq \frac{C_{1}}{2(r+1)}\left(\|u\|_{2(r+1)}^{2(r+1)}+\|v\|_{2(r+1)}^{2(r+1)}\right) \\
& \leq \frac{C_{1} B^{2(r+1)}}{2(r+1)}\left(\|\nabla u\|_{m}^{2(r+1)}+\|\nabla v\|_{m}^{2(r+1)}\right), \quad t \geq 0 .
\end{aligned}
$$

We obtain from (3.9) and (3.12) that

$$
\begin{aligned}
& a\left|\int_{\Omega}\left(\left|u_{t}\right|^{p-2} u_{t} u+\left|v_{t}\right|^{p-2} v_{t} v\right) d x\right| \\
& \quad \leq C_{3} H(t)^{\frac{1}{2(r+1)}-\frac{1}{p}}\left(\|u\|_{2(r+1)}^{\frac{2(r+1)}{p}}\left\|u_{t}\right\|_{p}^{p-1}+\|v\|_{2(r+1)}^{\frac{2(r+1)}{p}}\left\|v_{t}\right\|_{p}^{p-1}\right) .
\end{aligned}
$$

We get from (3.13), Lemma 2.2, Lemma 2.3 and (3.1) that

$$
\begin{aligned}
& a\left|\int_{\Omega}\left(\left|u_{t}\right|^{p-2} u_{t} u+\left|v_{t}\right|^{p-2} v_{t} v\right) d x\right| \\
& \quad \leq C_{3}\left[\varepsilon^{p}\left(\|u\|_{2(r+1)}^{2(r+1)}+\|v\|_{2(r+1)}^{2(r+1)}\right)+\varepsilon^{-\frac{p}{p-1}} H^{\prime}(t)\right] H(t)^{-\alpha},
\end{aligned}
$$

where $\alpha=\frac{1}{p}-\frac{1}{2(r+1)}, \varepsilon>0$. Let $0<\rho<\alpha$, then we have from (3.2) and (3.14) that

$$
\begin{aligned}
& a\left|\int_{\Omega}\left(\left|u_{t}\right|^{p-2} u_{t} u+\left|v_{t}\right|^{p-2} v_{t} v\right) d x\right| \\
& \quad \leq C_{3}\left[\varepsilon^{r} H(0)^{-\alpha}\left(\|u\|_{2(r+1)}^{2(r+1)}+\|v\|_{2(r+1)}^{2(r+1)}\right)+\varepsilon^{-\frac{p}{p-1}} H(0)^{\rho-\alpha} H(t)^{-\rho} H^{\prime}(t)\right] .
\end{aligned}
$$

Now, we define $L(t)$ as follows:

$$
L(t)=H(t)^{1-\rho}+\delta G(t), \quad t \geq 0,
$$


where $\delta$ is a positive constant to be determined later. By differentiating (3.16), we see from (3.8) and (3.15) that

$$
\begin{aligned}
L^{\prime}(t)= & (1-\rho) H(t)^{-\rho} H^{\prime}(t)+\delta G^{\prime}(t) \\
\geq & {\left[1-\rho-\delta C_{3} \varepsilon^{-\frac{p}{p-1}} H(0)^{\rho-\alpha}\right] H(t)^{-\rho} H^{\prime}(t) } \\
& +\delta\left[(r+2)\left(\left\|u_{t}\right\|^{2}+\left\|v_{t}\right\|^{2}\right)+2(r+1) H(t)\right] \\
& +\frac{2(r+1)-m}{m} \cdot \frac{\left(\lambda_{2}^{m}-\lambda_{1}^{m}\right)}{\lambda_{2}^{m}} \delta\left(\|\nabla u\|_{m}^{m}+\|\nabla v\|_{m}^{m}\right) \\
& -C_{3} \delta \varepsilon^{r} H(0)^{-\alpha}\left(\|u\|_{2(r+1)}^{2(r+1)}+\|v\|_{2(r+1)}^{2(r+1)}\right) .
\end{aligned}
$$

Letting $k=\min \left\{r+1, \frac{2(r+1)-m}{m} \cdot \frac{\left(\lambda_{2}^{m}-\lambda_{1}^{m}\right)}{\lambda_{2}^{m}}\right\}$ and decomposing $2 \delta(r+1) H(t)$ in (3.17) by

$$
2 \delta(r+1) H(t)=2 k \delta H(t)+2 \delta(r+1-k) H(t),
$$

we find from (2.4) that

$$
\int_{\Omega} F(u, v) d x \geq \frac{C_{0}}{2(r+1)}\left(\|u\|_{2(r+1)}^{2(r+1)}+\|v\|_{2(r+1)}^{2(r+1)}\right) .
$$

Combining (3.1)-(3.2) and (3.17)-(3.19), we obtain that

$$
\begin{aligned}
L^{\prime}(t) \geq & {\left[1-\rho-\delta C_{3} \varepsilon^{-\frac{p}{p-1}} H(0)^{\rho-\alpha}\right] H(t)^{-\rho} H^{\prime}(t) } \\
& +\delta[(r+2)-k]\left(\left\|u_{t}\right\|^{2}+\left\|v_{t}\right\|^{2}\right)+2 \delta(r+1-k) H(t) \\
& +\left[\frac{2(r+1)-m}{m} \cdot \frac{\left(\lambda_{2}^{m}-\lambda_{1}^{m}\right)}{\lambda_{2}^{m}}-k\right] \delta\left(\|\nabla u\|_{m}^{m}+\|\nabla v\|_{m}^{m}\right) \\
& +\left[\frac{k C_{0}}{r+1}-C_{3} \varepsilon^{p} H(0)^{-\alpha}\right] \delta\left(\|u\|_{2(r+1)}^{2(r+1)}+\|v\|_{2(r+1)}^{2(r+1)}\right) .
\end{aligned}
$$

Choosing $\varepsilon>0$ small enough such that $\varepsilon^{p}<\frac{k C_{0}}{(r+1) C_{3}} H(0)^{\alpha}$ and $0<\delta<\frac{1-\rho}{C_{3}} \varepsilon^{\frac{p}{p-1}} H(0)^{\alpha-\rho}$, we have from (3.20) that

$$
L^{\prime}(t) \geq C_{4} \delta\left[\left\|u_{t}\right\|^{2}+\left\|v_{t}\right\|^{2}+\|\nabla u\|_{m}^{m}+\|\nabla v\|_{m}^{m}+\|u\|_{2(r+1)}^{2(r+1)}+\|v\|_{2(r+1)}^{2(r+1)}+H(t)\right],
$$

where $C_{4}=\left\{r+2-k, 2(r+1-k), \frac{k C_{0}}{2(r+1)}, \frac{2(r+1)-m}{m} \cdot \frac{\left(\lambda_{2}^{m}-\lambda_{1}^{m}\right)}{\lambda_{2}^{m}}-k\right\}$. Therefore, $L(t)$ is a nondecreasing function for $t \geq 0$. Letting $\delta$ in (3.16) be small enough, we get $L(0)>0$. Consequently, we obtain that $L(t) \geq L(0)>0$ for $t \geq 0$.

Since $0<\rho<\alpha<1$, it is evident that $1<\frac{1}{1-\rho}<\frac{1}{1-\alpha}$. We deduce from (3.3) and (3.16) that

$$
L(t)^{\frac{1}{1-\rho}} \leq C_{5}\left[H(t)+\left(\delta \int_{\Omega}\left(u u_{t}+v v_{t}\right) d x\right)^{\frac{1}{1-\rho}}+\left(\delta\left[\|\nabla u(t)\|^{2}+\|\nabla v(t)\|^{2}\right]\right)^{\frac{1}{1-\rho}}\right] .
$$

On the other hand, for $r>0$, we have from Hölder's inequality and Lemma 2.2 that

$$
\begin{aligned}
\left(\delta \int_{\Omega}\left(u u_{t}+v v_{t}\right) d x\right)^{\frac{1}{1-\rho}} & \leq C_{6}\left(\left\|u_{t}\right\|^{\frac{1}{1-\rho}}\|u\|_{2(r+1)}^{\frac{1}{1-\rho}}+\left\|v_{t}\right\|^{\frac{1}{1-\rho}}\|v\|_{2(r+1)}^{\frac{1}{1-\rho}}\right) \\
& \leq C_{7}\left(\|u\|_{2(r+1)}^{\frac{\mu}{1-\rho}}+\|v\|_{2(r+1)}^{\frac{\mu}{1-\rho}}+\left\|u_{t}\right\|^{\frac{v}{1-\rho}}+\left\|v_{t}\right\|^{\frac{v}{1-\rho}}\right)
\end{aligned}
$$


where $\frac{1}{\mu}+\frac{1}{v}=1$. Let $0<\rho<\min \left\{\alpha, \frac{1}{2}-\frac{1}{2(r+1)}, 1-\frac{2}{m}\right\}, v=2(1-\rho)$, then $\frac{\mu}{1-\rho}=\frac{2}{1-2 \rho}<2(r+1)$. It follows from (3.12) that

$$
\begin{aligned}
& \left(\frac{C_{1}}{2(r+1) H(0)}\right)^{\frac{1}{2(r+1)}}\|u\|_{2(r+1)} \geq 1, \quad\left(\frac{C_{1}}{2(r+1) H(0)}\right)^{\frac{1}{2(r+1)}}\|v\|_{2(r+1)} \geq 1 \\
& \left(\frac{C_{1} B^{2(r+1)}}{2(r+1) H(0)}\right)^{\frac{1}{2(r+1)}}\|\nabla u\|_{m} \geq 1, \quad\left(\frac{C_{1} B^{2(r+1)}}{2(r+1) H(0)}\right)^{\frac{1}{2(r+1)}}\|\nabla v\|_{m} \geq 1 .
\end{aligned}
$$

Thus, we get from (3.24) that

$$
\begin{aligned}
& \|u\|_{2(r+1)}^{\frac{\mu}{1-\rho}}=\|u\|_{2(r+1)}^{\frac{2}{1-2 \rho}} \leq\left(\frac{C_{1}}{2(r+1) H(0)}\right)^{1-\frac{1}{(1-2 \rho)(r+1)}}\|u\|_{2(r+1)}^{2(r+1)}, \\
& \|v\|_{2(r+1)}^{\frac{\mu}{1-\rho}}=\|v\|_{2(r+1)}^{\frac{2}{1-2 \rho}} \leq\left(\frac{C_{1}}{2(r+1) H(0)}\right)^{1-\frac{1}{(1-2 \rho)(r+1)}}\|v\|_{2(r+1)}^{2(r+1)} .
\end{aligned}
$$

We obtain from (3.23) and (3.26) that

$$
\left(\delta \int_{\Omega}\left(u u_{t}+v v_{t}\right) d x\right)^{\frac{1}{1-\rho}} \leq C_{8}\left(\left\|u_{t}\right\|^{2}+\left\|v_{t}\right\|^{2}+\|u\|_{2(r+1)}^{2(r+1)}+\|v\|_{2(r+1)}^{2(r+1)}\right) .
$$

Similarly, we have from Hölder's inequality and (3.25) that

$$
\left(\delta\left[\|\nabla u\|^{2}+\|\nabla v\|^{2}\right]\right)^{\frac{1}{1-\rho}} \leq C_{9}\left(\|\nabla u\|_{m}^{\frac{2}{1-\rho}}+\|\nabla v\|_{m}^{\frac{2}{1-\rho}}\right) \leq C_{10}\left(\|\nabla u\|_{m}^{m}+\|\nabla v\|_{m}^{m}\right) .
$$

Combining (3.22), (3.27) and (3.28), we find that

$$
L(t)^{\frac{1}{1-\rho}} \leq C_{11}\left[\left\|u_{t}\right\|^{2}+\left\|v_{t}\right\|^{2}+\|\nabla u\|_{m}^{m}+\|\nabla v\|_{m}^{m}+\|u\|_{2(r+1)}^{2(r+1)}+\|v\|_{2(r+1)}^{2(r+1)}+H(t)\right] .
$$

We obtain from (3.21) and (3.29) that

$$
L^{\prime}(t) \geq C_{12} L(t)^{\frac{1}{1-\rho}}, \quad t \geq 0,
$$

where $C_{12}=\frac{C_{4} \delta}{C_{11}}$. Integrating both sides of (3.30) over $[0, t]$ yields

$$
L(t) \geq\left(L(0)^{\frac{\rho}{1-\rho}}-C_{12} \rho t\right)^{-\frac{\rho}{1-\rho}} .
$$

Note that $L(0)>0$, then there exists $T *=T_{\max }=\frac{(1-\rho) L(0)^{\frac{\rho}{1-\rho}}}{C_{12} \rho}$ such that $L(t) \rightarrow+\infty$ as $t \rightarrow$ $+\infty$. Namely, the solutions of problem (1.1)-(1.5) blow up in finite time.

Competing interests

The author declares that they have no competing interests.

\section{Acknowledgements}

This research was supported by the National Natural Science Foundation of China (No. 61273016), the Natural Science Foundation of Zhejiang Province (No. Y6100016), Zhejiang province universities scientific research key project (Z201017584). 


\section{References}

1. Segal, I: Nonlinear partial differential equation in quantum field theory. Proc. Symp. Appl. Math. 17, $210-226$ (1965)

2. Medeiros, LA, Miranda, MM: Weak solutions for a system of nonlinear Klein-Gordon equations. Ann. Mat. Pura Appl. CXLVI, 173-183 (1987)

3. Miranda, MM, Medeiros, LA: On the existence of global solutions of a coupled nonlinear Klein-Gordon equations. Funkc. Ekvacioj 30, 147-161 (1987)

4. da Silva Ferreira, J: Exponential decay of the energy of a nonlinear system of Klein-Gordon equations with localized damping in bounded and unbounded domains. Asymptot. Anal. 8, 73-92 (1994)

5. Cavalcanti, MM, Domingos Cavalcanti, VN, Prates Filho, JS, Soriano, JA: Existence and uniform decay of a degenerate and generalized Klein-Gordon system with boundary damping. Commun. Appl. Anal. 21, 65-83 (2002)

6. Komornik, V, Zuazua, E: A direct method for the boundary stabilization of the wave equation. J. Math. Pures Appl. 69 44-54 (1990)

7. Yang, ZJ: Existence and asymptotic behaviour of solutions for a class of quasilinear evolution equations with nonlinear damping and source terms. Math. Methods Appl. Sci. 25, 795-814 (2002)

8. Yang, ZJ, Chen, GW: Global existence of solutions for quasilinear wave equations with viscous damping. J. Math. Anal. Appl. 285, 604-618 (2003)

9. Yang, ZJ: Initial boundary value problem for a class of nonlinear strongly damped wave equations. Math. Methods Appl. Sci. 26, 1047-1066 (2003)

10. Liu, YC, Zhao, JS: Multidimensional viscoelasticity equations with nonlinear damping and source terms. Nonlinear Anal. 56, 851-863 (2004)

11. Yang, ZJ: Blow-up of solutions for a class of quasilinear evolution equations with nonlinear damping and source terms. Math. Methods Appl. Sci. 25, 825-833 (2002)

12. Messaoudi, SA, Said-Houari, B: Global nonexistence of solutions of a class of wave equations with nonlinear damping and source terms. Math. Methods Appl. Sci. 27, 1687-1696 (2004)

13. Messaoudi, SA: On the decay of solutions for a class of quasilinear hyperbolic equations with nonlinear damping and source terms. Math. Methods Appl. Sci. 28, 1819-1828 (2005)

14. Kavashima, S, Shibata, Y: Global existence and exponential stability of small solutions to nonlinear viscoelasticity. Commun. Math. Phys. 148, 189-208 (1992)

15. Haraux, A, Zuazua, E: Decay estimates for some semilinear damped hyperbolic problems. Arch. Ration. Mech. Anal. $150,191-206$ (1988)

16. Kopackova, M: Remark on bounded solutions of a semilinear dissipative hyperbolic equation. Comment. Math. Univ. Carol. 30, 713-719 (1989)

17. Ball, J: Remarks on blow up and nonexistence theorems for nonlinear evolutions equations. Q. J. Math. 28,473-486 (1977)

18. Wu, ST, Tsai, LY: On global solutions and blow-up of solutions for a nonlinearly damped Petrovsky system. Taiwan. J. Math. 13, 545-558 (2009)

19. Payne, LE, Sattinger, DH: Saddle points and instability of nonlinear hyperbolic equations. Isr. J. Math. 22, 273-303 (1975)

20. Vitillaro, E: Global nonexistence theorems for a class of evolution equations with dissipation. Arch. Ration. Mech. Anal. 149, 155-182 (1999)

21. Messaoudi, SA, Said-Houari, B: Global nonexistence of positive initial energy solutions of a system of nonlinear viscoelastic wave equations with damping and source terms. J. Math. Anal. Appl. 365, 277-287 (2010)

22. Gao, HJ, Ma, TF: Global solutions for a nonlinear wave equation with the $p$-Laplacian operator. Electron. J. Qual. Theory Differ. Equ. 1999, 11 (1999)

doi:10.1186/s13661-014-0251-y

Cite this article as: Ye: Global nonexistence of solutions for systems of quasilinear hyperbolic equations with damping and source terms. Boundary Value Problems 2014 2014:251.

\section{Submit your manuscript to a SpringerOpen ${ }^{\ominus}$ journal and benefit from:}

- Convenient online submission

Rigorous peer review

- Immediate publication on acceptance

Open access: articles freely available online

- High visibility within the field

- Retaining the copyright to your article 